
Copyright () 2016 World Federation for Ultrasound in Medicine \& Biology Printed in the USA. All rights reserved $0301-5629 / \$$ - see front matter

http://dx.doi.org/10.1016/j.ultrasmedbio.2016.06.024

\title{
FACTORS PREDICTIVE OF IMPROVED ABDOMINAL ULTRASOUND VISUALIZATION AFTER ORAL ADMINISTRATION OF SIMETHICONE
}

\author{
Maria Marsico, ${ }^{*}$ Tommaso Gabbani, ${ }^{\dagger}$ Tommaso Casseri, ${ }^{\ddagger}$ and Maria Rosa Biagini ${ }^{\dagger}$ \\ * Division of Gastroenterology, AOU Modena University Hospital, Modena, Italy; ${ }^{\dagger}$ Oncology Department, Division of \\ Gastroenterology, AOU Careggi University Hospital, Florence, Italy; and ${ }^{\ddagger}$ Division of Radiology, AOU Senese, Siena \\ University Hospital, Siena, Italy
}

(Received 21 December 2015; revised 2 June 2016; in final form 6 June 2016)

\begin{abstract}
Ultrasonography is a non-invasive, accurate and low-cost technique used to study the upper abdomen, but it has reduced reliability in the study of the pancreas and retroperitoneum. Simethicone is a well-known emulsifying agent that has been used to improve ultrasonographic visualization. The aim of this study was to identify anthropometric parameters that are able to predict a good response to simethicone in improving ultrasonographic visualization of abdominal structures. One hundred twenty-seven patients were recruited. After basal examination, their anthropometric parameters were collected. Patients with an incomplete upper abdominal examination because of gastrointestinal gas have greater body mass index, waist circumference and abdominal wall thickness. In our study, the best anthropometric parameter for identifying patients with poor visualization at abdominal ultrasound examination is waist circumference. Using a cutoff of $84 \mathrm{~cm}$, we can identify patients with poor visibility at abdominal ultrasound examination (group B) with a sensitivity of 90\%. (E-mail: tom.gabbani@gmail. com) (c) 2016 World Federation for Ultrasound in Medicine \& Biology.
\end{abstract}

Key Words: Simethicone, Abdominal ultrasound, Pre-medication, Ultrasound diagnostic accuracy.

\section{INTRODUCTION}

Ultrasonography (US) is a non-invasive, accurate and low-cost technique used to study the upper part of the abdomen, but it has a reduced reliability in the study of pancreas and retroperitoneum (Ishigami et al. 2014; Warren et al. 1978). The limitation during exploration of the pancreas is often attributed to meteorism caused by excess gas in the stomach or gut in majority of the cases. Several techniques have been used to improve pancreatic US imaging, but these techniques have had limited success (Rosenberg et al. 1982). If the visualization of the pancreas or other abdominal structures is poor, frequent computed tomography (CT) scan or a magnetic resonance imaging (MRI) should be performed although at a high health care cost. In recent years, many different pre-medications have been proposed to improve ultrasound imaging quality of the upper abdomen with good results. Authors have proposed the use of oral cellulosebased contrast medium (Harisinghani et al. 1997). Others

Address correspondence to: Tommaso Gabbani, Dipartimento Oncologico Gastroenterologia SOD 1, AOU Careggi, Largo Brambilla, 3-50139 Firenze, Italy. E-mail: tom.gabbani@gmail.com have suggested the administration of water (Lev-Toaff et al. 1999). Moreover, some authors have even proposed the use of enemas, provoking considerable discomfort for the patient without obtaining satisfactory results (Friis et al. 1991; Weighall et al. 1979). Several studies have reported improved visualization of abdominal structures, particularly the pancreas, using oral specific pre-medications with simethicone (Lund et al. 1992; Sisler and Tilcock 1995). Simethicone is a well-known emulsifying agent that has been used previously in upper gastrointestinal radiologic examinations to break down large gas pockets by changing their surface tension. It is also known to improve ultrasonographic visualization of the pancreas and the retroperitoneum in association with patient rotation (Abu-Yousef and El-Zein 2000; Herrerías-Gutiérrez and García-Montes 1994; Päivänsalo and Suramo 1986; Varas and López 1991). However, the extensive use of pre-medication for all patients who have to undergo abdominal ultrasound is not cost-effective and can subject some patients who do not need pre-medication to adverse events related to these pre-medications (Worlicek et al. 1989). The aim of this study was to identify anthropometric parameters that are able to predict a good response to simethicone oral 
administration in terms of improving the ultrasonographic visualization of pancreas, and other abdominal structures. We have also compared prospectively two different doses of oral simethicone to identify the best dose in terms of cost/benefit ratio to use as premedication for abdominal ultrasound.

\section{METHODS}

One hundred twenty-seven consecutive patients $>20$ and $<90$ y old, who came to our outpatient clinic to undergo abdominal ultrasound, were recruited. The study was approved by the relevant local ethics committee, and Declaration of Helsinki protocols were followed. Written informed consent was obtained from all patients. Each patient was examined with basal abdominal ultrasound. To quantify the visibility of the structures, we chose landmarks of interest (LOIs: pancreas head, body and tail; left hepatic lobe; caudate lobe; right and left kidney; abdominal aorta; splenic vein and spleen) that were used to calculate a LOI score. The latter has been evaluated by the sum of the visualized LOIs $(0=$ LOI not visible or $1=$ LOI visible). The maximum LOI score was defined as 10 points. After the basal ultrasound examination, the following anthropometric parameters of patients were collected: weight, height, waist circumference, abdominal wall thickness. Patients were undressed for these measurements. For each patient we used the same medical instrumentations (meter and scales) previously calibrated and provided by our hospital. Weight and height of patients were used to calculate the body mass index (BMI). Patients were divided in two groups: group A (34 patients) for patients with a complete abdominal ultrasonographic visualization (LOI score $=10$ ) and group B (93 patients) with an incomplete abdominal ultrasonographic visualization (LOI score $<10$ ). We excluded from the study patients who had eaten or smoked before the examination, who had not consumed the prescribed amount of water before the examination, who were unable to rotate three full turns on the examination bed and who could not stand upright if necessary. The 93 patients from group B were randomly divided into three subgroups. Patients in the first subgroup drank $15 \mathrm{~mL}$ of a simethicone solution $1 \mathrm{~h}$ before the US examination. Patients in the second subgroup drank a complete therapeutic dose of simethicone $(15 \mathrm{~mL} \times 2 / \mathrm{d})$ for 3 consecutive $\mathrm{d}$ before the examination. Patients in the third subgroup drank $15 \mathrm{~mL}$ of placebo solution $1 \mathrm{~h}$ before the examination. The placebo solution was a mixture of water, sucrose, thickener and lemon taste and was prepared to resemble, in taste and texture, the simethicone solution. The study was performed double blind; the simethicone or placebo solu- tion was delivered by the nurse. The examination was performed by two expert ultrasonographers with the same echograph, a ProSound Alfa7 (Hitachi-Aloka, Tokyo, Japan) with a 3.75- to 7.5-MHz UST 91-30 Multifrequency Convex Abdominal HST (Hemispheric Sound Technology) probe. The sonographers optimized the image for each patient by changing such parameters as the frequency of transmission, gain, time gain compensation and dynamic range during the scan to effectively select patients with poor visualization for the study. All examinations were performed at the fundamental frequency; tissue harmonic imaging (THI) was never used. The mechanical index was $<1.9$ as prescribed by U.S. Food and Drug Administration guidelines for ultrasound scanners. During the examination, each patient was rotated $180^{\circ}$ to the right and to the left three times, and only those for whom visualization was incomplete were re-examined in the upright position. The purpose of asking patients to rotate is to induce a gas bowel displacement to improve and maximize the visualization in agreement with what is reported in the literature (Abu-Yousef and El-Zein 2000; Herrerías-Gutiérrez and García-Montes 1994; Päivänsalo and Suramo 1986; Varas and López 1991). The US examination lasted between 20 and $30 \mathrm{~min}$. Abdominal wall thickness was estimated when performing the ultrasound measurement of the subcutaneous adipose tissue at the level of the xiphoid process. Data base construction and data analysis were performed using Office Excel 2007, XLSTAT 2016 (Addinsoft) and SPSS for Windows (IBM, Armonk, NY, USA). We examined the data with the use of appropriate parametric and non-parametric statistical tests (two-tailed Student $t$-test and Fischer's $\chi^{2}$ test, with $p<0.05$ indicating significance); the likelihood ratio and relative risk were calculated. A Lilliefors (Kolmogorov-Smirnov) test for normality had been performed earlier. With the degree of visualization of the abdomen (complete group A or incomplete group B) as a benchmark, sensitivity and specificity were calculated for two definitions of waist circumference: (i) greater than the cutoff value obtained and (ii) less than the cutoff value. Sensitivity was calculated as the number of patients with incomplete ultrasound visualization and waist circumference greater than the cutoff value. Specificity was calculated as the number of patients with complete ultrasound visualization and waist circumference less than the cutoff value. Receiver operating characteristic (ROC) curves was plotted, and the areas under the ROC curves (AUCs) were calculated along with their $95 \%$ confidence intervals (CIs). The 95\% CIs were used to compare diagnostic capabilities. The cutoff was calculated with ROC curve and ASC analysis (Hanley and McNeil 1982). 
Table 1. Main features of the groups

\begin{tabular}{|c|c|c|c|c|c|}
\hline \multirow{2}{*}{$\begin{array}{c}\text { Anthropometric parameters } \\
\text { of patients }\end{array}$} & \multirow{2}{*}{$\begin{array}{l}\text { Group A } \\
(\mathrm{n}=34)\end{array}$} & \multicolumn{3}{|c|}{ Group B } & \multirow{2}{*}{$\begin{array}{l}p \text { Value (group } \\
\text { A/group B) }\end{array}$} \\
\hline & & Subgroup $1(\mathrm{n}=39)$ & Subgroup $2(\mathrm{n}=38)$ & Subgroup $3(n=16)$ & \\
\hline Age, $y$ & $52.9 \pm 16.7$ & $55.6 \pm 18.1$ & $57.2 \pm 17.9$ & $56.4 \pm 14.9$ & $>0.05$ \\
\hline Body mass index, $\mathrm{kg} / \mathrm{m}^{2}$ & $23.23 \pm 2.89$ & $26.04 \pm 4.09$ & $26.74 \pm 4.49$ & $25.75 \pm 3.82$ & $>0.05$ \\
\hline Waist circumference, $\mathrm{cm}$ & $84 \pm 9.8$ & $93.2 \pm 13.4$ & $97.1 \pm 11.6$ & $87.8 \pm 12.5$ & $>0.05$ \\
\hline Abdominal wall thickness, $\mathrm{cm}$ & $2.03 \pm 0.61$ & $2.50 \pm 1.04$ & $2.66 \pm 1.09$ & $2.36 \pm 0.74$ & $>0.05$ \\
\hline
\end{tabular}

\section{RESULTS}

One hundred twenty-seven consecutive patients (68 men, 59 women; 59 , age: $56.4 \pm 16.7$ y [mean \pm standard deviation]; BMI: $26.27 \pm 4.24 \mathrm{~kg} / \mathrm{m}^{2}$; waist circumference: $93.9 \pm 12.7 \mathrm{~cm}$; abdominal wall thickness: $2.54 \pm 0.99 \mathrm{~cm}$ ) who had undergone upper abdominal US examination were enrolled in this study (Table 1). There were 34 patients in group A (complete visualization of the abdomen) and 93 patients in group B (incomplete visualization of the abdomen) (Fig. 1a, b). In group $\mathrm{B}$, the mean global visibility at the basal ultrasound examination was 5.82 (LOI score). After randomization, 39 patients were included in subgroup 1, 38 patients in subgroup 2 and 16 patients in subgroup 3. In subgroups 1 and $2(\mathrm{n}=77)$, the mean global visibility was 5.66 at the basal (first) ultrasound examination (subgroup 1: 6, subgroup 2: 5.27), whereas in subgroup 3, the mean global visibility was 6.69. In group B (incomplete visualization of the abdomen), at the basal ultrasound examination, the pancreas was not fully viewable in 91 cases $(97.8 \%)$. In particular, the head was not visualized in 44 cases $(48.4 \%)$, the body in 69 cases $(75.8 \%)$ and the tail in 90 cases $(98.9 \%)$. In subgroup 1, the pancreas was not completely viewable in 38 cases $(97.4 \%)$, and the head was not visualized in 15 cases (39.5\%), the pancreas body in 30 cases $(78.9 \%)$ and the pancreas tail in 38 cases $(100 \%)$. In subgroup 2, the pancreas was not completely viewable in 37 cases $(97.4 \%)$, and the head was not visualized in 25 cases $(67.6 \%)$, the body in 30 cases $(81.1 \%)$ and the tail in 36 cases $(67.3 \%)$. In subgroup 3, the pancreas was not completely viewable in all patients $(100 \%)$, and the head was not visualized in $4(25 \%)$, the body in $9(56.3 \%)$ and the tail in $16(100 \%)$. In group $\mathrm{B}$ (incomplete visualization of the abdomen), the left lobe or the caudate lobe of the liver was not viewable in 26 cases $(27.9 \%)$, the right kidney in 3 cases $(3.2 \%)$, the left kidney in only 1 case $(1.1 \%)$, the abdominal aorta in 30 cases (34.1\% of cases) and the splenic vein in 26 cases $(32.3 \%)$. In all cases, the spleen was correctly visualized. Mean global visibility at the second ultrasound examination was 8.13 for subgroup $1,7.76$ for subgroup 2 and 7.07 for subgroup 3 . The pancreas was not fully viewable in 50 cases (53.8\%). In subgroup 1, the pancreas was not completely viewable in 20 cases (51.3\%); in subgroup 2 , in 18 cases $(47.4 \%$ ); and in subgroup 3, in 12 cases $(75 \%)$. At the second US examination, the left lobe of the liver was not viewable in 2 cases $(2.2 \%)$, the abdominal aorta in 12 cases (12.9\% of cases) and the splenic vein in 10 cases $(10.8 \%)$. The spleen, right kidney and
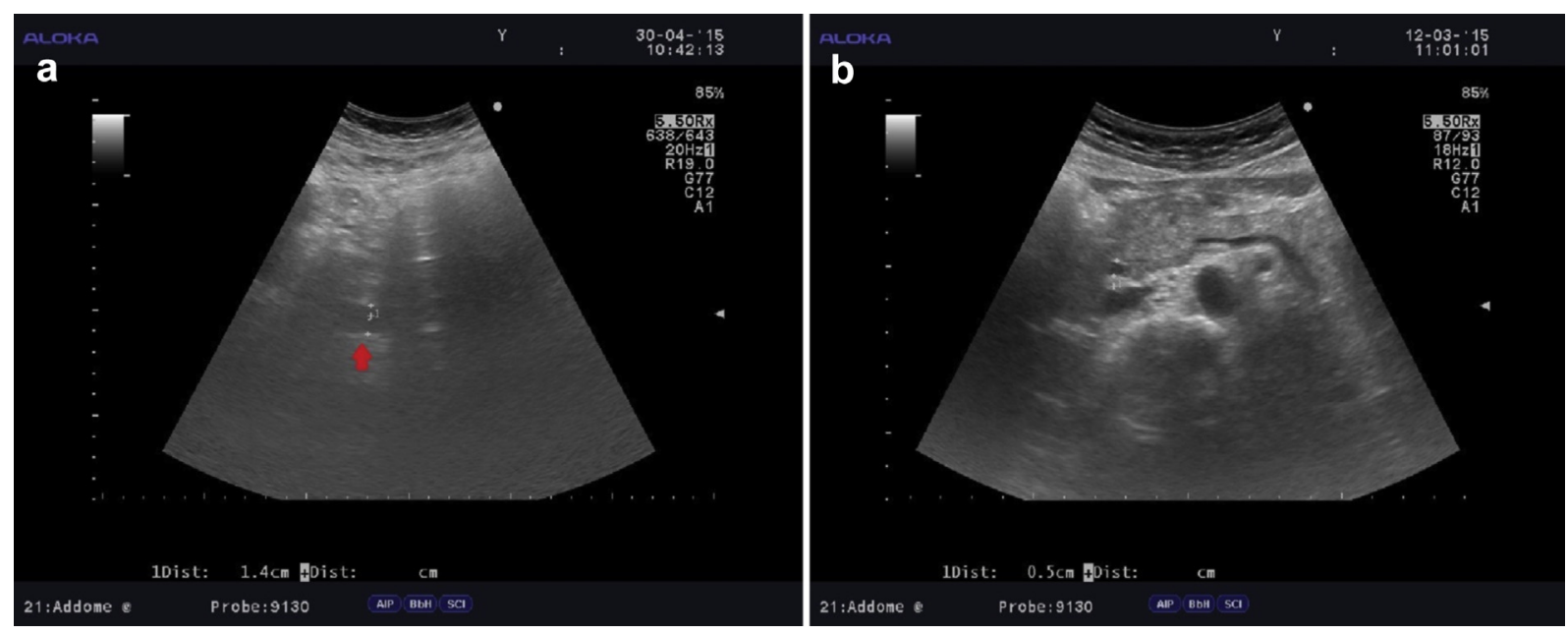

Fig. 1. (a) Pancreas and epigastric structures not viewable for inter-position of abdominal gas before administration of simethicone. The red arrow indicates the splenic vein. (b) Viewable pancreas after simethicone administration. 
Landmarks of Interest score

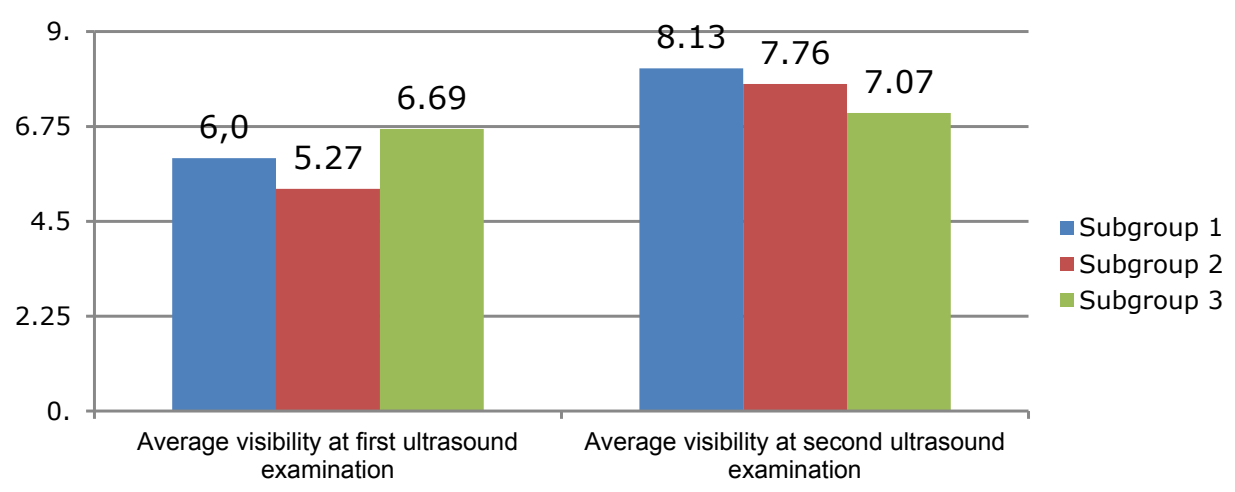

Fig. 2. Global visibility at the first and second ultrasonographic (US) examinations. $p$ Values are reported in the text.

left kidney were visualized in all cases. Global visibility calculated with the LOI score improved significantly after administration of simethicone solution $(p<0.01)$ in subgroups 1 and 2 . There was no significant improvement in global visibility in subgroup 3 (placebo) $(p>0.05)$. In particular, visualization of the pancreas improved significantly in subgroups 1 and $2(p<0.01)$, but not in subgroup $3(p>0.05)$. At the second examination, the LOI score was significantly higher in subgroups 1 and 2 with respect to subgroup 3 (placebo) $(p<0.01)$. No statistical difference in global visualization (LOI score) was observed between the two different dosages of simethicone solution (subgroups 1 and 2). Global and pancreas visualization increased respectively from $37.6 \%$ to $44.7 \%$ at US examination after the administration of simethicone. LOIs were completely visualized (score at second US examination $=9$ ) in 36 patients $(38.7 \%)$. There was $41.6 \%$ improvement in global US visualization

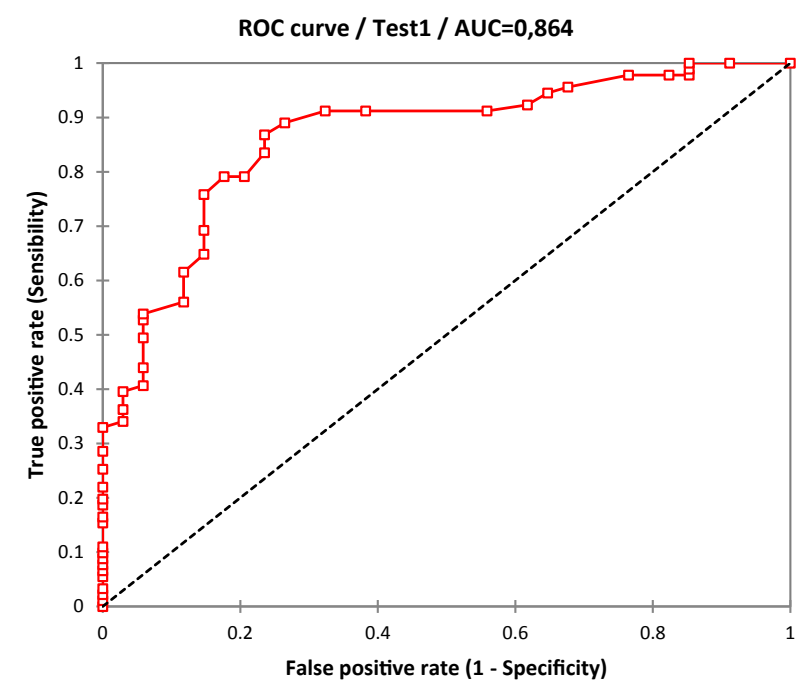

Fig. 3. ROC curve. ROC $=$ receiver operating characteristic; $\mathrm{AUC}=$ area under the ROC curve. in subgroups 1 and 2 together. Complete visualization of the pancreas at the second US examination was achieved in 41 patients $(45.1 \%)$. The main visibility characteristics of the different subgroups are illustrated in Figure 2. In group A, the mean global visibility at the basal ultrasound examination was the highest possible in LOI score. There were 34 patients in this group (11 males and 23 females) (age: $53.1 \pm 15.8 \mathrm{y}$; BMI: $22.56 \pm 3.43 \mathrm{~kg} / \mathrm{m}^{2}$; waist circumference: $83.2 \pm 9.6 \mathrm{~cm}$; abdominal wall thickness: $1.93 \pm 0.70)$. There were some statistically significant differences between groups A and B with respect to BMI, waist circumference and abdominal wall thickness. In particular, patients from group B were found to have higher BMIs $(p<0.01)$, greater waist circumference $(p<0.01)$ and thicker abdominal walls $(p<0.01)$ in comparison with group A. In the total study population, the sensitivity for incomplete visualization of the abdomen at a waist circumference cutoff value of $84 \mathrm{~cm}$ was $90 \%$ (95\% CI: $0.807-0.940$ ), and specificity was $73.5 \%$ (95\% CI: 0.566-0.855). The AUC for waist circumference was 0.864 (95\% CI: 0.801-0.927) (Fig. 3).

\section{DISCUSSION}

Ultrasonography is an important method for studying the upper abdomen; nevertheless, the presence of gas in the stomach and gut is the main reason for poor visualization of important structures. Ultrasonographic artifacts during air inter-position prevent visualization of the underlying structures, resulting in the use of other expensive diagnostic imaging techniques. Visualization of the pancreas, in particular, is the major limitation. To increase the visibility of the upper abdominal region, medications that avoid the inter-position of air present in the gastrointestinal lumen can be used in ultrasonography. Simethicone is a carminative agent that can emulsify foam and air bubbles in the gastrointestinal tract by decreasing their superficial tension and permitting their 
coalescence. Simethicone is a mixture of polydimethylsiloxane and hydrated silica gel. It is not absorbed by the gut into the bloodstream and is therefore, considered relatively tolerable. The National Institutes of Health (NIH) reports there are usually no side effects with oral simethicone. Contraindications are allergy to the active substance and intestinal obstruction. Small intestine bacterial overgrowth syndrome (SIBO) is one of the most common causes of excessive abdominal gas. According to the literature, already SIBO is commonly associated with many pathologic conditions such as advanced chronic liver disease (Chesta and Defilippi 1993; Stotzer et al. 1996), obesity (Madrid et al. 1997; Tosetti et al. 1996), irritable bowel syndrome (Pimentel et al. 2003; Posserud et al. 2007) and probably hepatic steatosis (Sabaté et al. 2008; Wittmann et al. 2010). Moreover, we know that patients with common structural disorders (such as diverticula) or functional disorders (alteration of microbiota) of the colon are predisposed to the development of gut bacterial overgrowth and excessive abdominal gas (Brecević et al. 1994; Goldstein and Katragadda 1978; Madrid et al. 2011). An association between SIBO or colonic disorders and obesity has been already reported (Di Stefano et al. 2005; Skar et al. 1989; Vantrappen et al. 1977). So, we believe that patients with larger waist circumferences and overweight, based on the BMI, have excessive bowel gas, probably because of SIBO or colonic structural and functional disorders. However, it is certain that a larger waist circumference is related to a deeper pancreas and more abdominal and mesenteric fat, which can negatively influence ultrasound visualization, but simethicone probably cannot influence this condition. According to the available literature, our trial has indicated that pre-medication with simethicone can improve the diagnostic capability of upper abdominal ultrasonography, especially for pancreas visualization, excluding the use of other expensive examinations such as CT and MRI (Foster et al. 1984; Hessel et al. 1982; Kamin et al. 1980; Pasanen et al. 1992). In our study, we compared the benefits of two different types of administration of simethicone as pre-medication for ultrasound examination. We compared standard therapeutic doses of simethicone for 3 consecutive days before the examination with a single simethicone administration $1 \mathrm{~h}$ before the ultrasound examination. We found that pre-treatment with oral simethicone solution can improve the accuracy of US upper abdomen examination $(p<0.01)$, with an increase of $41.6 \%$ in global US visibility. The single dose results in better dosage in terms of the cost/benefit ratio. In addition, we found that patients with the worst ultrasound visualization caused by gastrointestinal gas (group B) have greater BMI, waist circumference and abdominal wall thickness in comparison with group A (patients with complete visualization). In our study, the best anthropometric parameter for identifying patients with a poor visualization at abdominal ultrasound examination is waist circumference. Using a cutoff of $84 \mathrm{~cm}$, we can identify patients with poor visibility at abdominal ultrasound examination (group B) with a sensitivity of $90 \%$. Therefore, we propose that a few simple anthropometric predictive factors could identify patients who should routinely receive simethicone before abdominal ultrasound. We conclude that values higher than normal for BMI, abdominal wall thickness and, in particular, waist circumference are predictive of the presence of excessive intestinal gas, which results in worse visualization of the upper abdomen at ultrasound examination. For this reason, we propose that it would be useful to routinely prescribe simethicone before abdominal ultrasound to patients with high values of these anthropometric parameters, particularly waist circumference $>84 \mathrm{~cm}$. Finally, we suggest that a single dose of simethicone administered $1 \mathrm{~h}$ before the ultrasound examination is the best pre-medication in terms of the cost/benefit ratio.

\section{REFERENCES}

Abu-Yousef MM, El-Zein Y. Improved US visualization of the pancreatic tail with simethicone, water, and patient rotation. Radiology 2000;217:780-785.

Brecević L, Bosan-Kilibarda I, Strajnar F. Mechanism of antifoaming action of simethicone. J Appl Toxicol 1994;14:207-211.

Chesta J, Defilippi C. Abnormalities in proximal small bowel motility in patients with cirrhosis. Hepatology 1993;17:828-832.

Di Stefano M, Miceli E, Missanelli A, Mazzocchi S, Corazza GR. Absorbable vs. non-absorbable antibiotics in the treatment of small intestine bacterial overgrowth in patients with blind-loop syndrome. Aliment Pharmacol Ther 2005;21:985-992.

Foster PN, Mitchell CJ, Robertson DRC, Hamilton I, Irving $\mathrm{H}$ Kelleher J, Lintott DJ, Robinson PJ, Axon AT, Losowsky MS. Prospective comparison of three non-invasive tests for pancreatic disease. Br Med J 1984;289:13-16.

Friis H, Bodé S, Rumessen JJ, Gudmand-Høyer E. Effect of simethicone on lactulose-induced $\mathrm{H}_{2}$ production and gastrointestinal symptoms. Digestion 1991;49:227-230.

Goldstein HM, Katragadda CS. Prone view ultrasonography for pancreatic tail neoplasms. AJR Am J Roentgenol 1978;131:231-234.

Hanley JA, McNeil BJ. The meaning and use of the area under a receiver operating characteristic (ROC) curve. Radiology 1982;143:29-36.

Harisinghani MG, Saini S, Schima W, McNicholas M, Mueller PR. Simethicone coated cellulose as an oral contrast agent for ultrasound of the upper abdomen. Clin Radiol 1997;52:224-226.

Herrerías Gutiérrez JM, García Montes J. The effect of clebopride-simethicone combination therapy on echographic visualization of retrogastric organs. Clin Ther 1994;16:483-489.

Hessel SJ, Siegelman SS, McNeil BJ, Sanders R, Adams DF, Alderson PO, Finberg HJ, Abrams HL. A prospective evaluation of computed tomography and ultrasound of the pancreas. Radiology 1982;143:129-133.

Ishigami K, Abu-Yousef DM, Kao SC, Abu-Yousef MM. Comparison of 2 oral ultrasonography contrast agents: Simethicone-coated cellulose and simethicone-water rotation in improving pancreatic visualization. Ultrasound Q 2014;30:135-138.

Kamin PD, Bernardino ME, Wallace S, Jing BS. Comparison of ultrasound and computed tomography in the detection of pancreatic malignancy. Cancer 1980;46:2410-2412. 
Lev-Toaff AS, Langer JE, Rubin DL, Zelch JV, Chong WK, Barone AE, Goldberg BB. Safety and efficacy of a new oral contrast agent for sonography: A phase II trial. AJR Am J Roentgenol 1999;173:431-436.

Lund PJ, Fritz TA, Unger EC, Hunt RK, Fuller E. Cellulose as a gastrointestinal US contrast agent. Radiology 1992;185:783-788.

Madrid AM, Cumsille F, Defilippi C. Altered small bowel motility in patients with liver cirrhosis depends on severity of liver disease. Dig Dis Sci 1997;42:738-742.

Madrid AM, Poniachik J, Quera R, Defilippi C. Small intestinal clustered contractions and bacterial overgrowth: a frequent finding in obese patients. Dig Dis Sci 2011;56:155-160.

Päivänsalo M, Suramo I. Ultrasonography of the pancreatic tail through spleen and through fluid-filled stomach. Eur J Radiol 1986;6:113-115.

Pasanen P, Partanen K, Pikkarainen P, Alhava E, Pirinen A, Janatuinen E. Diagnostic accuracy of ultrasound, computed tomography and endoscopic retrograde cholangiopancreatography in the detection of pancreatic cancer in patients with jaundice or cholestasis. In Vivo 1992;6:297-302.

Pimentel M, Chow EJ, Lin HC. Normalization of lactulose breath testing correlates with symptom improvement in irritable bowel syndrome: A double-blind, randomized, placebo-controlled study. Am J Gastroenterol 2003;98:412-419.

Posserud I, Stotzer PO, Björnsson ES, Abrahamsson H, Simrén M. Small intestinal bacterial overgrowth in patients with irritable bowel syndrome. Gut 2007;56:802-808.

Rosenberg ER, Clair MR, Bowie JD. The fluid-filled stomach as an acoustic window to the left kidney. AJR Am J Roentgenol 1982; 138:175-176.

Sabaté JM, Jouët P, Harnois F, Mechler C, Msika S, Grossin M, Coffin B. High prevalence of small intestinal bacterial overgrowth in patients with morbid obesity: A contributor to severe hepatic steatosis. Obes Surg 2008;18:371-377.
Sisler WJ, Tilcock C. Effect of cellulose concentration on the efficacy of a cellulose-based oral contrast agent for gastrointestinal ultrasonography. J Ultrasound Med 1995;14:267-272.

Skar V, Skar AG, Osnes M. The duodenal bacterial flora in the region of papilla of Vater in patients with and without duodenal diverticula. Scand J Gastroenterol 1989;24:649-656.

Stotzer PO, Björnsson ES, Abrahamsson H. Interdigestive and postprandial motility in small-intestinal bacterial overgrowth. Scand J Gastroenterol 1996;31:875-880.

Tosetti C, Corinaldesi R, Stanghellini V, Pasquali R, Corbelli C, Zoccoli G, Di Febo G, Monetti N, Barbara L. Gastric emptying of solids in morbid obesity. Int J Obes Relat Metab Disord 1996;20: 200-205.

Vantrappen G, Janssens J, Hellemans J, Ghoos Y. The interdigestive motor complex of normal subjects and patients with bacterial overgrowth of the small intestine. J Clin Invest 1977;59: $1158-1166$

Varas MJ, López A. Improved image quality during abdominal ultrasonography by clebopride + simethicone. Methods Find Exp Clin Pharmacol 1991;13:69-72.

Warren PS, Garrett WJ, Kossoff G. The liquid-filled stomach—An ultrasonic window to the upper abdomen. J Clin Ultrasound 1978;6: 315-320.

Weighall SL, Wolfman NT, Watson N. The fluid-filled stomach: A new sonic window. J Clin Ultrasound 1979;7:353-356.

Wittmann T, Paradowski L, Ducrott P, Bueno L, Andro Delestrain MC. Clinical Trial: The efficacy of alverine citrate/simeticone combination on abdominal pain/discomfort in irritable bowel syndrome- $\mathrm{A}$ randomized, double-blind, placebo-controlled study. Aliment Pharmacol Therap 2010;31:615-624.

Worlicek H, Dunz D, Engelhard K. Ultrasonic examination of the wall of the fluid-filled stomach. J Clin Ultrasound 1989;17:5-14. 